\title{
Dynamical correlations in financial systems
}

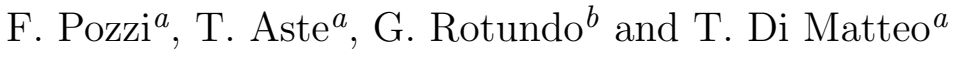 \\ ${ }^{a}$ Department of Applied Mathematics, The Australian National University, 0200 Canberra, \\ ACT, Australia. \\ ${ }^{b}$ Department of Mathematics for Economic, Financial and Insurance Decisions, University of \\ Rome La Sapienza, Via del Castro Laurenziano, 9 Rome, 00161, Italy.
}

\begin{abstract}
One of the main goals in the field of complex systems is the selection and extraction of relevant and meaningful information about the properties of the underlying system from large datasets. In the last years different methods have been proposed for filtering financial data by extracting a structure of interactions from cross-correlation matrices where only few entries are selected by means of criteria borrowed from network theory. We discuss and compare the stability and robustness of two methods: the Minimum Spanning Tree and the Planar Maximally Filtered Graph. We construct such graphs dynamically by considering running windows of the whole dataset. We study their stability and their edges's persistence and we come to the conclusion that the Planar Maximally Filtered Graph offers a richer and more significant structure with respect to the Minimum Spanning Tree, showing also a stronger stability in the long run.
\end{abstract}

Keywords: Econophysics; Complex Systems; Networks; Minimum Spanning Tree; Planar Maximally Filtered Graph; Financial Data Correlations.

\section{INTRODUCTION}

In the last few years, many filtering methods have been developed by econophysicists in order to extract relevant information from huge amount of financial data. Two of such methods are the Minimum Spanning Tree (denoted from now on as $M S T)^{1}$ used by Mantegna for financial data in ref. ${ }^{2}$ and the Planar Maximally Filtered Graph (denoted from now on as $P M F G$ ) introduced by Tumminello et al. in ref. ${ }^{3}$

In this paper we analyze, compare and discuss the robustness, the stability and the structural fluctuations of $M S T$ and $P M F G$ considered as graphs dynamically evolving over time.

This paper is organized as follows. In section 2 we illustrate the data set and we introduce the correlation matrices and the associated complete graphs from financial time series. We show that there are some similarities between dynamical systems of correlations built on moving dynamical windows of different lengths $\Delta t$ and the static system built on the entire data set which can be seen as its long run stable structure. In section 3 the dynamical MST and PMFG are introduced and described, and some properties of such subgraphs are discussed and compared with the dynamical complete graphs from which they are extracted. In section 4 we discuss differences in the averages and in standard deviations computed over subgraphs and complete graphs. A comparison with systems of interest rates has been made and it shows significant differences which are directly associated to the specific peculiarities of the markets. In order to assess the robustness of such graphs, in section 5 the frequencies of appearance of edges in the dynamical MSTs and PMFGs are computed for each given $\Delta t$. In section 6 we introduce a new graph built as the union of all edges that can be reached with a $T 1$ elementary movement from a given $P M F G \cdot^{4,5}$ Intersections between dynamical subgraphs and their corresponding static subgraphs are computed and relevant differences related to long-run time-persistence of edges are shown. In section 7 we draw some conclusions and propose some suggestions for future research.

Send correspondence to frp110@rsphysse.anu.edu.au

Complex Systems II, edited by Derek Abbott, Tomaso Aste, Murray Batchelor, Robert Dewar,

Tiziana Di Matteo, Tony Guttmann, Proc. of SPIE Vol. 6802, 68021E, (2008)

0277-786X/08/\$18 - doi: $10.1117 / 12.758822$

Proc. of SPIE Vol. $680268021 \mathrm{E}-1$ 


\section{DYNAMICAL CORRELATIONS}

\subsection{Data description}

We have analyzed daily time series of the $n=300$ most capitalized $N Y S E$ companies from 2001 to 2003, for a total of $T=748$ days. Return time series are computed as logarithmic differences of daily prices, and daily prices are computed as averages of daily quotations. Closing quotations are excluded from the computation. In the following we denote with $Y$ the $748 \times 300$ matrix of returns.

Stocks are classified into 12 economic sectors and 77 economic subsectors.

\subsection{Distance Matrices from correlations}

Let us consider all time data subsets of dimensions $\Delta t \times 300$, where $\Delta t$ corresponds to a moving window, from time $(t)$ to time $(t+\Delta t-1)$, where $t=1,2, \ldots,(T-\Delta t+1)$ and $\Delta t=21,42,63,84,126,251$ days, corresponding approximately to $\Delta t=1,2,3,4,6,12$ months. For each $t$ and $\Delta t$, the resulting matrix is denoted as $Y_{\tau, s}$ with $\tau=t,(t+1), \ldots,(t+\Delta t-1)$ and $s=1,2, \ldots, 300$.

The number of these matrices, for each choice of $\Delta t$, is shown in Table 1.

Table 1. Number of dynamical correlation matrices associated to the choice of the moving window $\Delta t$.

\begin{tabular}{||c||c||c||}
\hline $\begin{array}{c}\Delta \mathbf{t} \\
\text { months }\end{array}$ & $\begin{array}{c}\Delta \mathbf{t} \\
\text { days }\end{array}$ & $\begin{array}{c}\text { cases } \\
\mathbf{n}^{\mathbf{o}}\end{array}$ \\
\hline 1 & 21 & 728 \\
\hline 2 & 42 & 707 \\
\hline 3 & 63 & 686 \\
\hline 4 & 84 & 665 \\
\hline 6 & 126 & 623 \\
\hline 12 & 251 & 498 \\
\hline 36 & 748 & 1 \\
\hline \hline
\end{tabular}

For each of such matrices, we computed the correlation matrix $C(t, \Delta t)$, which is a $300 \times 300$ matrix with coefficients given by the formula

$$
c_{i, j}(t, \Delta t)=\frac{\left\langle Y_{\tau, i} Y_{\tau, j}\right\rangle_{\tau}-\left\langle Y_{\tau, i}\right\rangle_{\tau}\left\langle Y_{\tau, j}\right\rangle_{\tau}}{\sqrt{\left(\left\langle Y_{\tau, i}^{2}\right\rangle_{\tau}-\left\langle Y_{\tau, i}\right\rangle_{\tau}^{2}\right)\left(\left\langle Y_{\tau, j}^{2}\right\rangle_{\tau}-\left\langle Y_{\tau, j}\right\rangle_{\tau}^{2}\right)}}
$$

where $\left\langle f_{\tau}\right\rangle_{\tau}=\frac{1}{\Delta t} \sum_{\tau=1}^{\Delta t} f_{\tau}$ is the time average of a given series $f_{\tau}$. From the correlation coefficients $c_{i, j}$, we can write a well-known measure of distance between stocks $i$ and $j: d_{i, j}=\sqrt{2\left(1-c_{i, j}\right)}$. Such distance is the euclidean metric distance computed between standardized returns $Z_{\tau, i}$ of stocks $i$ and $j$ where

$$
Z_{\tau, i}=\frac{Y_{\tau, i}-\left\langle Y_{\tau, i}\right\rangle_{\tau}}{\sqrt{\left(\left\langle Y_{\tau, i}^{2}\right\rangle_{\tau}-\left\langle Y_{\tau, i}\right\rangle_{\tau}^{2}\right)}}
$$

The distance $d_{i, j}$ is a function, $d: Y_{\tau, i} \times Y_{\tau, j} \rightarrow R$, such that $d_{i, j} \in[0,2]$, with $d_{i, j}=0$ if $c_{i, j}=1, d_{i, j}=\sqrt{2}$ if $c_{i, j}=0$ and $d_{i, j}=2$ if $c_{i, j}=-1$. All standard properties of a metric distance are satisfied.

The matrices $D(t, \Delta t)=\sqrt{2(1-C(t, \Delta t))}$ can be interpreted as dynamical distance matrices of weighted complete graphs $K_{300}$ where all 300 stocks are interconnected. 

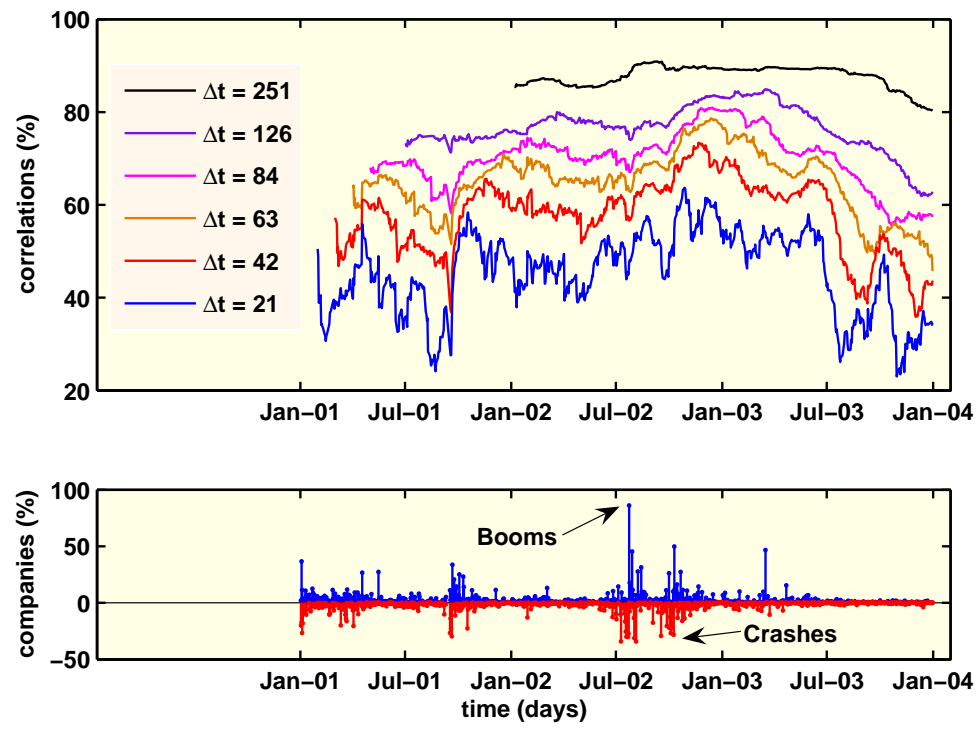

Figure 1. Correlations between the dynamical distance matrices $D(t, \Delta t)$, computed at $\Delta t=21,42,63,84,126,251$ days, corresponding approximately to $1,2,3,4,6,12$ months, and the static distance matrix $D^{*}$ obtained by using all $T=748$ days available from data set of the 300 most capitalized NYSE stocks' time series, corresponding to years $2001-2003$. The higher curve is obtained for $\Delta t=251$ days, the lower for $\Delta t=21$ days. At the bottom, percentages of companies whose standardized return, at each time $t$, exceeds two standard deviations or falls below minus two standard deviations.

\subsection{Static Graph and Dynamical Graphs}

As a first step we computed the static distance matrix $D^{*}$ on the entire data set $Y$ and, for each $t$ and $\Delta t$, we computed the correlations between such matrix and the dynamical distance matrices $D(t, \Delta t)$. Such correlations are

$$
E(t, \Delta t)=\frac{\left\langle d_{i, j}(t, \Delta t) d_{i, j}^{*}\right\rangle_{i, j}-\left\langle d_{i, j}(t, \Delta t)\right\rangle_{i, j}\left\langle d_{i, j}^{*}\right\rangle_{i, j}}{\sqrt{\left(\left\langle d_{i, j}^{2}(t, \Delta t)\right\rangle_{i, j}-\left\langle d_{i, j}(t, \Delta t)\right\rangle_{i, j}^{2}\right)\left(\left\langle d_{i, j}^{* 2}\right\rangle_{i, j}-\left\langle d_{i, j}^{*}\right\rangle_{i, j}^{2}\right)}}
$$

with $d_{i, j}$ and $d_{i, j}^{*}$ respectively the elements of the distance matrices $D(t, \Delta t)$ and $D^{*}$ and where $\left\langle f_{i, j}\right\rangle_{i, j}=$ $\frac{2}{n(n-1)} \sum_{i<j} f_{i, j}$ denotes the average of $f_{i, j}$ over all edges.

Correlations $E(t, \Delta t)$ for each $t$ and $\Delta t$ are shown in Figure 1. At the bottom of the figure, we show the percentage of companies whose standardized return, at each time $t$, exceeds two standard deviations (proxy measure for booms, positive values) or falls below minus two standard deviations (proxy measure for crashes, negative values).

As $\Delta t$ increases, we observe that the dynamical distance matrices get at every step closer to the static distance matrix, built on the entire data set. Relevant fluctuations observed at low levels of $\Delta t$, turn out to be strongly damped at higher levels. We observe that, after periods of particular turbulence, the dynamical system of correlations becomes closer to the static distance matrix. As we can see from Table 2 , when $\Delta t=21$ days, the range of correlations is from a minimum of $23.02 \%$ to a maximum of $63.67 \%$, the average being $45.39 \%$ and standard deviation $8.86 \%$. When $\Delta t=84$ days, the range of correlations is from a minimum of $55.47 \%$ to a maximum of $80.94 \%$, the average being $69.95 \%$ and standard deviation $6.08 \%$. When $\Delta t=251$ days, the range of correlations is from a minimum of $80.28 \%$ to a maximum of $90.93 \%$, the average being $87.73 \%$ and standard deviation $2.34 \%$. Thus, we see that the range becomes progressively narrower and the average higher. 
Table 2. Summary for correlations between static distance matrix $D^{*}$ and the dynamical distance matrices $D(t, \Delta t)$.

\begin{tabular}{||c||c||c||c||c||}
\hline \hline $\boldsymbol{\Delta t}$ & Min & Mean & Max & Std \\
\hline \hline 21 & 0.2302 & 0.4539 & 0.6367 & 0.0886 \\
42 & 0.3588 & 0.5778 & 0.7342 & 0.0803 \\
63 & 0.4573 & 0.6510 & 0.7865 & 0.0683 \\
84 & 0.5547 & 0.6995 & 0.8094 & 0.0608 \\
126 & 0.6188 & 0.7664 & 0.8493 & 0.0492 \\
251 & 0.8028 & 0.8773 & 0.9093 & 0.0234 \\
\hline \hline
\end{tabular}

It's worth mentioning that dynamical distance matrices built on only one third of the entire data set (corresponding to $\Delta t=251$ days) are very close to the static distance matrix built on the entire data set. This is showing a fast convergence with $\Delta t$ of the dynamical distances towards the static distances.

\section{DYNAMICAL MINIMUM SPANNING TREES AND PLANAR MAXIMALLY FILTERED GRAPHS}

The graphs associated to matrices $D(t, \Delta t)$ are complete graphs $K_{300}$, which have $n(n-1) / 2=(300)(299) / 2=$ 44850 edges connecting all pairs of nodes. Different methods exist in literature in order to filter such a huge amount of data, otherwise hardly readable and usable. One approach consists in extracting a sub-graph which retains the most valuable information and eliminates most of the redundancies, producing identifiable hierarchies and communities.

A widely used method is the Minimum Spanning Tree $(M S T)$, used for the first time in finance literature by Mantegna. $^{2}$ The $M S T$ is a tree, a graph with no cycles, in which all nodes are connected, and edges are selected in order to minimize the sum of distances. The total number of edges is $n-1$, where $n$ is the number of nodes. Several algorithms to construct the $M S T$ have been developed by the community of computer scientists and are widely known since 1926 (Otakar Boruvka's Algorithm). The most commonly used are Prim and Kruskal algorithms that find the $M S T$ in polynomial time. The efficiency of algorithms for finding the MST has been continuously enhanced over years (see, for instance, Eisner ${ }^{6}$ ). An almost linear running time algorithm has been recently developed by Chazelle. ${ }^{7}$ Since we have computed almost 4,000 MSTs out of 300 nodes's graphs, the efficiency of the algorithm had to be considered. We have used Prim's algorithm implemented in Matlab and we have found it efficient enough for our purposes.

A filtering method which uses a similar principle, but allows more interactions and a more complex and rich structure, is the Planar Maximally Filtered Graph ( $P M F G$ ), proposed for the first time by Tumminello et al. in ref. ${ }^{3}$ Such method constructs a connected planar graph ${ }^{8}$ where edges are selected in order to minimize the sum of distances. In this case, the total number of edges is $3(n-2)$, approximately the triple number of edges than the $M S T$. It has been proved by Tumminello et al. in ref. ${ }^{3}$ that the $M S T$ is always a subgraph of the PMFG. For each dynamical distance matrix $D(t, \Delta t)$ we computed the corresponding dynamical $M S T$ s and PMFGs. We computed also the Static $M S T$ and $P M F G$, over the entire period, in order to be able to compare their properties with those of the dynamical sub-graphs.

Averages and standard deviations have been computed for each $t$ and $\Delta t$ for edges belonging to the complete graphs $D(t, \Delta t)$, to the dynamical $M S T(t, \Delta t)$ and to the dynamical $P M F G(t, \Delta t)$. Moreover, for each $t$ and $\Delta t$ we computed the averages and standard deviations for edges belonging to the Static MST and $P M F G$. The average distances in the dynamically moving distance matrices for all the graphs, computed at $\Delta t=21$ days and at $\Delta t=251$ days are shown in Figure 2.

We observe that the average of complete graphs's distances can be considered as a superior limit: dynamical $M S T$ s and $P M F G$ s must have average distances lower or equal than the corresponding complete graphs. Conversely, distance averages of edges belonging to the Static MST and PMFG can be higher: but if this happens it indicates the total lack of significance and robustness for the relative subgraphs's selection. 

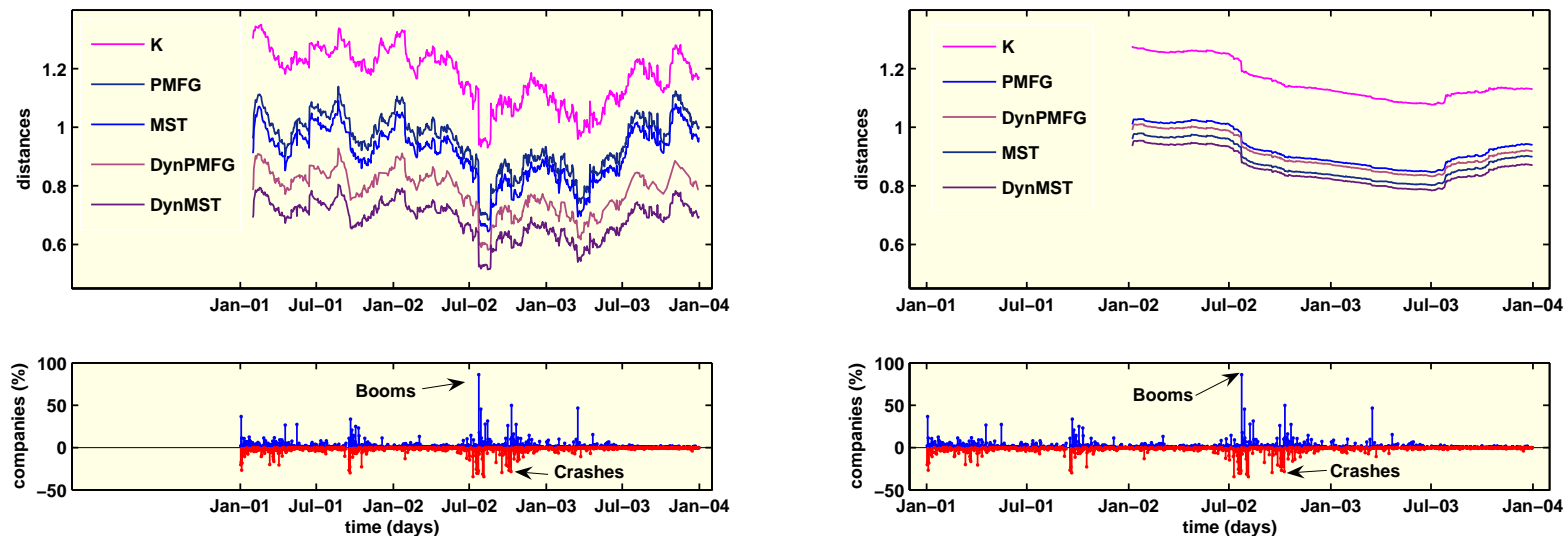

Figure 2. From top to bottom, on the left: the average distances in the dynamically moving distance matrices $D(t, \Delta t)$ computed at $\Delta t=21$ days, for edges belonging to: the complete graph, the Static $P M F G$ computed on all 748 days, the Static MST computed on all 748 days, the dynamical PMFGs computed at $\Delta t=21$ days, the dynamical $M S T$ s computed at $\Delta t=21$ days. On the right: $\Delta t=251$ days; averages of the dynamical $P M F G$ s are above those of the Static $M S T$. At the bottom, percentages of companies whose standardized return, at each time $t$, exceeds two standard deviations or falls below minus two standard deviations.

When $\Delta t=21$ days, the figure can be divided in three regions: at the top average distances of complete graphs's edges; in the middle average distances of the edges belonging to the Static PMFG and MST; at the bottom average distances of dynamical $P M F G$ s and MSTs. All curves show the same patterns and trends: subgraphs MSTs and $P M F G$ s reproduce well the properties of their corresponding complete graphs.

When $\Delta t=251$ days, the figure can be divided in two regions only: at the top average distances of the complete graphs; at the bottom, well beneath the first curve, average distances of edges belonging to the Static $P M F G$; then the dynamical $P M F G$; and further down the Static $M S T$ followed by the dynamical $M S T$. Note that in this case, the Static $M S T$ is below the dynamical $P M F G$.

Dynamical PMFGs exhibit behaviors and performances thoroughly similar to dynamical MSTs, with only slightly higher average distances.

It is of some interest to note that a remarkable sudden fall, consequent to turbulences due to the July/October 2002 stock market downturn, is clearly visible and is protracted for the entire period $\Delta t$ (21 days in the first case, 251 days in the second) and after that it is suddenly and completely re-absorbed. It is noteworthy that a single anomalous data point in July 2002 influences the average distances for all $\Delta t$ following periods.

We observe that the average distances of the dynamical graphs $M S T$ and $P M F G$ are closer to average distances of edges belonging to the corresponding static graphs than to the complete graph, with tightening gaps as $\Delta t$ increases. This means that the selection of edges performed by our graph's filtering is significantly robust.

\section{THE MEAN- $\sigma$ PLANE}

For each set of edges of the dynamical graphs and for each set of edges belonging to the static graphs, we calculated both the average distance and the standard deviation $\sigma$ in the matrices $D(t, \Delta t)$. We then consider the mean- $\sigma$ plane finding that, when $\Delta t=21$ days, variances of subgraphs's edges are almost always lower than or equal to variances of complete graphs's edges while, conversely, when $\Delta t=251$ days, variances of subgraphs edges are always higher.

For each time $t$, at a given $\Delta t$, each dynamical graph or each static edge selection is represented by one point in the mean- $\sigma$ plane. When $\Delta t=21$ days, these points in the mean- $\sigma$ plane are distributed uniformly on elliptical clouds, with each subgraph quite clustered. Differently, when $\Delta t=251$ days, the elliptical clouds become smooth straight lines, now very well distinct from each other, and their slopes are negative. Increasing 

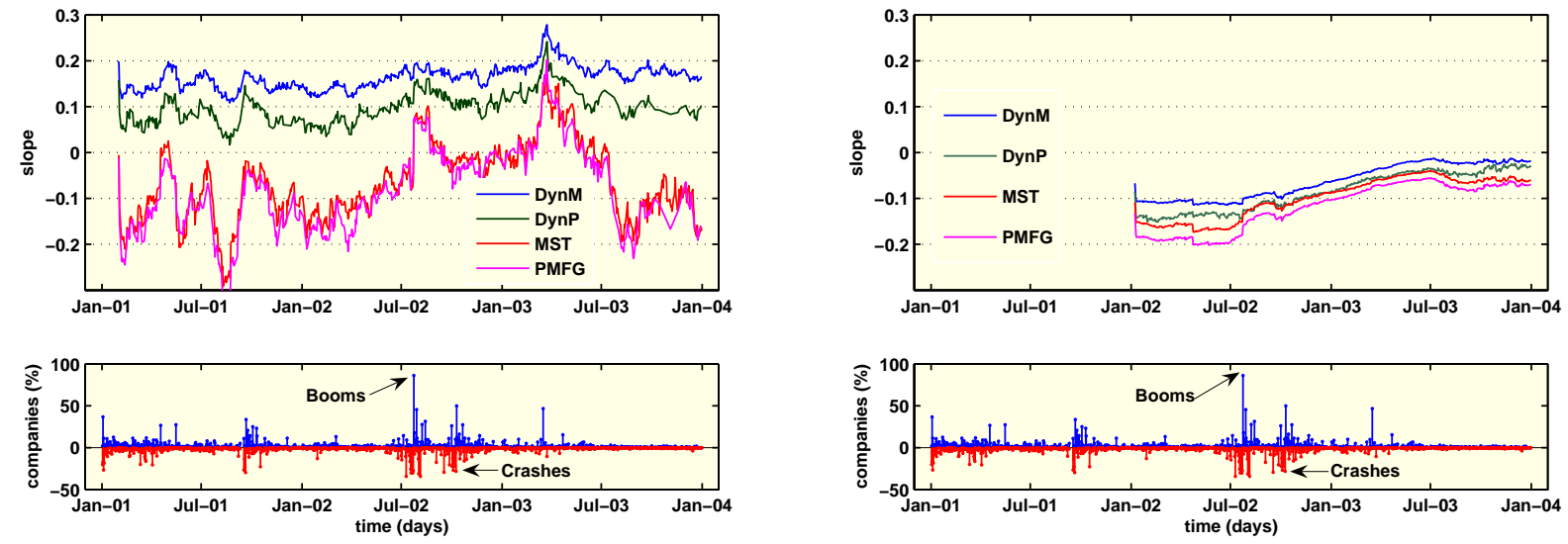

Figure 3. From top to bottom, slope in the mean- $\sigma$ plane of the lines joining one mean- $\sigma$ point of the dynamical complete graphs's edges to one mean- $\sigma$ point of edges belonging to the dynamical $M S T$ and $P M F G$, and to the Static $M S T$ and $P M F G$. On the left: $\Delta t=21$; on the right: $\Delta t=251$. At the bottom, percentages of companies whose standardized return, at each time $t$, exceeds two standard deviations or falls below minus two standard deviations.

$\Delta t$, if the average distance increases then the variance decreases and viceversa. We also computed the slope of the line connecting, at each time $t$, one mean- $\sigma$ point of all edges (complete graphs's) to one mean- $\sigma$ point of the dynamical $M S T$ or $P M F G$ or the Static $M S T$ or PMFG. We find that, when $\Delta t=21$ days (Figure 3 , left side), the slopes between the complete graph and the dynamical $M S T$ or $P M F G$ are always positive. Conversely the slopes between the complete graph and the Static $M S T$ or $P M F G$ are almost always negative, except for periods of particularly intense turbulence. On the other hand, when $\Delta t=251$ days (Figure 3, right side), the same slopes are always negative for all cases and for all periods. These findings imply that the system of 300 stocks is generally poorly correlated, with relatively small variances. But, during and after periods of intense turbulence, the time series get suddenly correlated and the variances increase.

We have made the same computations for a system of 16 Eurodollar interest rates's daily quotations and one more system of 34 interest rates's weekly quotations. ${ }^{9-14}$ We obtain in both cases the opposite result: a positive slope on the mean- $\sigma$ plane. This is not surprising because these are highly regulated systems indeed, monitored under strict control and strongly influenced by an international institutional system made of many cooperating national Central Banks. During a turbulent period, interest rates time series get partially decorrelated until agents and authorities adjust their positions, and then the system gets correlated again. Conversely, stock markets are highly competitive, hardly controllable, with dynamics hardly manageable and predictable, so they are much more complex and turbulent systems. During calm periods, the system is less correlated than during turbulent ones, when agents are driven by euphoria or panic; in such a system, public authorities have a lower control.

\section{FREQUENCIES OF SUB-GRAPHS'S EDGES}

Both $M S T$ and $P M F G$ select many statistically significant edges with high positive correlations but also some residual edges with lower weights. The dynamical graphs have some edges which appear often and others that are inserted only rarely. In order to detect significant edges, a frequency has been computed for each edge and for each $\Delta t$.

Both relative and absolute edge frequencies for dynamical MSTs are shown in Table 3 and Table 4, analogously for dynamical $P M F G$ s in Table 5 and Table 6.

We find that, when $\Delta t=251$ days, that is when the filtering is particularly robust, several edges that never appear in the dynamical MSTs appear very often (more than $70 \%$ of cases) in the dynamical PMFGs instead. We observe that more than $99.90 \%$ of all edges for dynamical MSTs and more than $99.50 \%$ of all edges for 
Table 3. Relative frequencies, at each $\Delta t$, for edges belonging to the dynamical $M S T \mathrm{~s}$.

\begin{tabular}{|c|c|c|c|c|c|c|c|c|}
\hline$\Delta t$ & $=0$ & $<0.1$ & $<0.2$ & $\cdots$ & $<0.7$ & $<0.8$ & $<0.9$ & $=1$ \\
\hline 1 & $42.22 \%$ & $99.31 \%$ & $99.81 \%$ & $\ldots$ & $99.99 \%$ & $99.99 \%$ & $100 \%$ & $0.00 \%$ \\
\hline 2 & $66.68 \%$ & $98.92 \%$ & $99.59 \%$ & $\ldots$ & $99.98 \%$ & $99.99 \%$ & $99.99 \%$ & $0.00 \%$ \\
\hline 3 & $77.73 \%$ & $98.68 \%$ & $99.44 \%$ & $\ldots$ & $99.98 \%$ & $99.98 \%$ & $99.99 \%$ & $0.01 \%$ \\
\hline 4 & $83.62 \%$ & $98.49 \%$ & $99.34 \%$ & $\ldots$ & $99.96 \%$ & $99.98 \%$ & $99.99 \%$ & $0.01 \%$ \\
\hline 6 & $89.45 \%$ & $98.25 \%$ & $99.18 \%$ & $\ldots$ & $99.93 \%$ & $99.95 \%$ & $99.98 \%$ & $0.01 \%$ \\
\hline 12 & $95.48 \%$ & $98.37 \%$ & $98.96 \%$ & $\ldots$ & $99.81 \%$ & $99.86 \%$ & $99.92 \%$ & $0.03 \%$ \\
\hline
\end{tabular}

Table 4. Absolute frequencies, at each $\Delta t$, for edges belonging to the dynamical $M S T$ s.

\begin{tabular}{|c|c|c|c|c|c|c|c|c|}
\hline$\Delta \mathrm{t}$ & $=0$ & $>0.1$ & $>0.2$ & $\cdots$ & $>0.7$ & $>0.8$ & $>0.9$ & $=1$ \\
\hline 1 & 18,936 & 310 & 85 & $\ldots$ & 5 & 4 & 2 & 0 \\
\hline 2 & 29,907 & 485 & 182 & $\ldots$ & 7 & 6 & 5 & 2 \\
\hline 3 & 34,864 & 593 & 250 & $\ldots$ & 11 & 7 & 6 & 3 \\
\hline 4 & 37,505 & 675 & 295 & $\ldots$ & 18 & 11 & 6 & 5 \\
\hline 6 & 40,120 & 785 & 370 & $\ldots$ & 33 & 23 & 9 & 6 \\
\hline 12 & 42,821 & 731 & 466 & $\ldots$ & 84 & 61 & 36 & 14 \\
\hline
\end{tabular}

Table 5. Relative frequencies, at each $\Delta t$, for edges belonging to the dynamical $P M F G$ s.

\begin{tabular}{||c||c||c||c||c||c||c||c||c||}
\hline \hline $\boldsymbol{\Delta}$ & \multicolumn{1}{|c||}{$\mathbf{0}$} & $<\mathbf{0 . 1}$ & $<\mathbf{0 . 2}$ & $\cdots$ & $<\mathbf{0 . 7}$ & $<\mathbf{0 . 8}$ & $<\mathbf{0 . 9}$ \\
& & & & & & & \\
\hline 1 & $8.76 \%$ & $97.65 \%$ & $99.03 \%$ & $\ldots$ & $99.97 \%$ & $99.98 \%$ & $99.99 \%$ & $0.00 \%$ \\
\hline \hline 2 & $20.58 \%$ & $96.93 \%$ & $98.5 \%$ & $\ldots$ & $99.88 \%$ & $99.92 \%$ & $99.96 \%$ & $0.00 \%$ \\
\hline 3 & $34.38 \%$ & $96.33 \%$ & $98.21 \%$ & $\ldots$ & $99.81 \%$ & $99.88 \%$ & $99.94 \%$ & $0.01 \%$ \\
\hline 4 & $44.74 \%$ & $95.92 \%$ & $98.04 \%$ & $\ldots$ & $99.74 \%$ & $99.84 \%$ & $99.92 \%$ & $0.02 \%$ \\
\hline 6 & $58.82 \%$ & $95.61 \%$ & $97.72 \%$ & $\ldots$ & $99.58 \%$ & $99.75 \%$ & $99.85 \%$ & $0.06 \%$ \\
\hline 12 & $79.14 \%$ & $95.56 \%$ & $97.21 \%$ & $\ldots$ & $99.23 \%$ & $99.44 \%$ & $99.63 \%$ & $0.19 \%$ \\
\hline \hline
\end{tabular}

Table 6. Absolute frequencies, at each $\Delta t$, for edges belonging to the dynamical PMFGs.

\begin{tabular}{|c|c|c|c|c|c|c|c|c|}
\hline$\Delta t$ & $=0$ & $>0.1$ & $>0.2$ & $\cdots$ & $>0.7$ & $>0.8$ & $>0.9$ & $=1$ \\
\hline 1 & 3,931 & 1,054 & 435 & $\ldots$ & 15 & 9 & 3 & 0 \\
\hline 2 & 9,232 & 1,377 & 673 & $\ldots$ & 54 & 36 & 16 & 2 \\
\hline 3 & 15,420 & 1,646 & 803 & $\ldots$ & 86 & 52 & 26 & 5 \\
\hline 4 & 20,068 & 1,828 & 879 & $\ldots$ & 115 & 73 & 37 & 11 \\
\hline 6 & 26,379 & 1,967 & 1,024 & $\ldots$ & 189 & 113 & 69 & 25 \\
\hline 12 & 35,495 & 1,992 & 1,252 & $\ldots$ & 347 & 249 & 166 & 84 \\
\hline
\end{tabular}


Table 7. Dynamical $M S T$ and $P M F G$ edges, with $100 \%$ frequency. $\Delta t=251$ days.

\begin{tabular}{||c||c||c||c||c||c||c||}
\hline \hline $\mathbf{i}$ & CODE & SECTOR & SUBSECTOR & CODE & SECTOR & SUBSECTOR \\
\hline \hline 1 & $S B C$ & Services & CommunicationServices & BLS & Services & CommunicationServices \\
\hline 2 & $F N M$ & Financial & ConsumFinancServ & $F R E$ & Financial & ConsumFinancServ \\
\hline 3 & $L E H$ & Financial & InvestmentServices & BSC & Financial & InvestmentServices \\
\hline 4 & $M B I$ & Financial & InsProp.\&Casualty & $A B K$ & Financial & InsProp.\&Casualty \\
\hline 5 & NEM & BasicMaterials & Gold\&Silver & ABX & BasicMaterials & Gold\&Silver \\
\hline 6 & $R D$ & Energy & Oil\&Gas - Integrated & TOT & Energy & Oil\&Gas - Integrated \\
\hline $\mathbf{7}$ & WLP & Financial & InsAccidental\&Health & HMA & Healthcare & HealthcareFacilities \\
\hline 8 & $L I Z$ & ConsumerCyclical & Apparel/Accessories & VFC & ConsumerCyclical & Apparel/Accessories \\
\hline 9 & CTX & CapitalGood & ConstructionServices & PHM & CapitalGood & ConstructionServices \\
\hline $\mathbf{1 0}$ & JP & Financial & InsLife & TMK & Financial & InsAccidental\&Health \\
\hline 11 & BJS & Energy & OilWellServ\&Equip & SII & Energy & OilWellServ\&Equip \\
\hline 12 & $K R I$ & Services & Printing\&Publishing & DJ & Services & Printing\&Publishing \\
\hline 13 & WLP & Financial & InsAccidental\&Health & $H U M$ & Financial & InsAccidental\&Health \\
\hline 14 & WHR & ConsumerCyclical & Appliance\&Tool & $M Y G$ & ConsumerCyclical & Appliance\&Tool \\
\hline \hline
\end{tabular}

Table 8. Dynamical PMFG edges, with high frequencies for PMFGs and $0 \%$ frequency for $M S T$ s. $\Delta t=251$ days.

\begin{tabular}{|c|c|c|c|c|c|c|c|}
\hline i & CODE & SECTOR & SUBSECTOR & CODE & SECTOR & SUBSECTOR & PMFG \\
\hline 1 & $G C I$ & Services & Printing\&Publishing & $D J$ & Services & Printing\&Publishing & 0.996 \\
\hline 2 & $S P G$ & Services & RealEstateOperations & $D R E$ & Services & RealEstateOperations & 0.9738 \\
\hline 3 & $B L S$ & Services & Communicationservices & $C T L$ & Services & Communicationservices & 0.9698 \\
\hline 4 & $A B K$ & Financial & InsProp.\&Casualty & $J P$ & Financial & InsLife & 0.9054 \\
\hline 5 & $M C D$ & Services & Restaurants & $E A T$ & Services & Restaurants & 0.8672 \\
\hline 6 & UTX & Conglomerates & Conglomerates & GD & CapitalGood & Aerospace\&Defense & 0.8672 \\
\hline 7 & $P F E$ & Healthcare & MajorDrugs & $A B T$ & Healthcare & MajorDrugs & 0.8632 \\
\hline 8 & $M B I$ & Financial & InsProp.\&Casualty & $T M K$ & Financial & InsAccidental\&Health & 0.8491 \\
\hline 9 & HMA & Healthcare & HealthcareFacilities & HUM & Financial & InsAccidental\&Health & 0.8491 \\
\hline 10 & $I P$ & BasicMaterials & Paper\&PaperProducts & $P P G$ & BasicMaterials & ChemicalManifacturing & 0.8471 \\
\hline 11 & MAS & ConsumerCyclical & Furniture\&Fixtures & CTX & CapitalGood & ConstructionServices & 0.829 \\
\hline 13 & $V F C$ & ConsumerCyclical & Apparel/Accessories & $J N Y$ & ConsumerCyclical & Apparel/Accessories & 0.7928 \\
\hline 14 & $I P$ & BasicMaterials & Paper\&PaperProducts & $P D$ & BasicMaterials & MetalMining & 0.7928 \\
\hline 15 & $C M A$ & Financial & RegionalBanks & $U P C$ & Financial & $N \backslash A$ & 0.7928 \\
\hline 16 & $P X$ & BasicMaterials & ChemicalManifacturing & $\mathrm{ROH}$ & BasicMaterials & Chemical-Plastic\&Rubber & 0.7827 \\
\hline 17 & $R D$ & Energy & Oil\&Gas - Integrated & $K M G$ & Energy & Oil\&GasOperations & 0.7807 \\
\hline 18 & $G G P$ & Services & RealEstateOperations & $D R E$ & Services & RealEstateOperations & 0.7746 \\
\hline 19 & GIS & ConsNonCycl & FoodProcessing & $C P B$ & ConsNonCycl & FoodProcessing & 0.7485 \\
\hline 20 & $B R$ & Energy & Oil\&GasOperations & $U C L$ & Energy & Oil\&GasOperations & 0.7163 \\
\hline 21 & $O X Y$ & Energy & Oil\&GasOperations & $T O T$ & Energy & Oil\&Gas - Integrated & 0.7163 \\
\hline 22 & $N S M$ & Technology & Semiconductors & $L S I$ & Technology & Semiconductors & 0.7143 \\
\hline 23 & $M H P$ & Services & Printing\&Publishing & $D J$ & Services & Printing\&Publishing & 0.7022 \\
\hline
\end{tabular}

dynamical PMFGs have persistence lower than $80 \%$. More than $99 \%$ of all edges for dynamical $M S T$ s and more than $97 \%$ of all edges for dynamical $P M F G$ s have persistence lower than $20 \%$. It is noteworthy to observe that, when $\Delta t=21$ days, $42.2 \%$ of all edges for dynamical $M S T$ s but only $8.8 \%$ of all edges for dynamical PMFGs never appear. While, when $\Delta t=251$ days, $95.5 \%$ of all edges for dynamical $M S T$ s and $79.1 \%$ of all edges for dynamical PMFGs never appear. It is also remarkable that, when $\Delta t=21$ days, $99.3 \%$ of all edges for dynamical MSTs and $97.7 \%$ of all edges for dynamical PMFGs are selected in less than $10 \%$ of cases.

The most significant dynamical MST and PMFG edges, with 100\% frequencies, are shown in Table 7 . We notice that all edges identify a specific economic activity: in the large majority of cases, the two nodes belong to the same sector and sub-sector, and when this is not so, as in rows 7 and 10, the two activities are strictly related in a specific economic sense (ie. Insurance Accidental \& Health linked to Healthcare Facilities in the first case, Insurance Accidental \& Health linked to Insurance Life in the second case).

In Table 8 some of the most significant edges are shown which are often selected by $P M F G$ s (with a frequency of more than 70\%) but never selected by MSTs. All edges are, again, strictly associated to a specific economic activity: in most of them the two nodes belong to the same sector and sub-sector. When this is not so, as in rows 6, 9 and 11, the two activities are strictly related in a specific economic sense: UTX "provides a broad range of high-technology products and support services to customers in the aerospace and building industries" and it is linked to $G D$ that is an industry specialized in "Aerospace design, Combat Systems, Marine Systems design, Information Systems and Technology". Similarly, row 9 links the same sectors and subsectors as row 7 of Table 7. Analogously, $M A S$ is in the field of "Furniture \& Fixtures (faucets, kitchen, bath cabinets, bath and shower units, spas and hot tubs, shower and plumbing specialties, electronic lock sets and other builders' hardware, air treatment products, ventilating equipment and pumps)" and it is linked to home building company $C T X$ whose main field is "Construction Services" and whose "principal activities are to provide residential and 
Table 9. Dynamical MST and PMFG edges, with $100 \%$ frequency for PMFGs and different frequencies for $M S T$ s. $\Delta t=251$ days.

\begin{tabular}{|c|c|c|c|c|c|c|c|}
\hline $\mathbf{i}$ & CODE & SECTOR & SUBSECTOR & CODE & SECTOR & SUBSECTOR & MST \\
\hline 1 & $P P G$ & BasicMaterials & ChemicalManifacturing & $W Y$ & BasicMaterials & Forestry\&WoodProducts & 0.004 \\
\hline 2 & $T X N$ & Technology & Semiconductors & $N S M$ & Technology & Semiconductors & 0.012 \\
\hline 3 & $S L B$ & Energy & OilWellServ\&Equip & $B J S$ & Energy & OilWellServ\&Equip & 0.0141 \\
\hline 4 & $O X Y$ & Energy & Oil\&GasOperations & $U C L$ & Energy & Oil\&GasOperations & 0.0703 \\
\hline 5 & $S B C$ & Services & CommunicationServices & $A T$ & Services & CommunicationServices & 0.1044 \\
\hline 6 & $A H C$ & Energy & Oil\&Gas - Integrated & $K M G$ & Energy & Oil\&GasOperations & 0.1225 \\
\hline 7 & $M E R$ & Financial & InvestmentServices & $A G E$ & Financial & InvestmentServices & 0.1687 \\
\hline 8 & $B N I$ & Transportation & Railroad & $\mathrm{NSC}$ & Transportation & Railroad & 0.1888 \\
\hline 9 & $G C I$ & Services & Printing\&Publishing & $T R B$ & Services & Printing\&Publishing & 0.241 \\
\hline 10 & $P P G$ & BasicMaterials & ChemicalManifacturing & $P X$ & BasicMaterials & ChemicalManifacturing & 0.2671 \\
\hline 11 & MME & Healthcare & $\mathbf{N} \backslash \mathbf{A}$ & HUM & Financial & InsAccidental\&Health & 0.2871 \\
\hline 13 & $B H I$ & Energy & OilWellServ\&Equip & $B J S$ & Energy & OilWellServ\&Equip & 0.3253 \\
\hline 14 & $M E R$ & Financial & InvestmentServices & $B S C$ & Financial & InvestmentServices & 0.3414 \\
\hline 15 & $P P G$ & BasicMaterials & ChemicalManifacturing & $A P D$ & BasicMaterials & ChemicalManifacturing & 0.3514 \\
\hline 16 & $M B I$ & Financial & InsProp.\&Casualty & $M T G$ & Financial & InsProp.\&Casualty & 0.3735 \\
\hline 17 & $U N P$ & Transportation & Railroad & $\mathrm{NSC}$ & Transportation & Railroad & 0.3835 \\
\hline 18 & $D D$ & BasicMaterials & Chemical-Plastic\&Rubber & $P P G$ & BasicMaterials & ChemicalManifacturing & 0.4157 \\
\hline 19 & $S L B$ & Energy & OilWellServ\&Equip & SII & Energy & OilWellServ\&Equip & 0.4438 \\
\hline 20 & $E Q R$ & Services & RealEstateOperations & $A I V$ & Services & RealEstateOperations & 0.4839 \\
\hline 21 & $U N P$ & Transportation & Railroad & $B N I$ & Transportation & Railroad & 0.5141 \\
\hline 22 & $U N P$ & Transportation & Railroad & $C S X$ & Transportation & Railroad & 0.5402 \\
\hline 23 & $A P A$ & Energy & Oil\&GasOperations & $K M G$ & Energy & Oil\&GasOperations & 0.5422 \\
\hline 26 & $T R B$ & Services & Printing\&Publishing & $K R I$ & Services & Printing\&Publishing & 0.5622 \\
\hline 27 & $F P L$ & Utilities & ElectricUtilities & $C I N$ & Utilities & ElectricUtilities & 0.5703 \\
\hline 28 & $M B I$ & Financial & InsProp.\&Casualty & $J P$ & Financial & InsLife & 0.5723 \\
\hline 29 & $I P$ & BasicMaterials & Paper\&PaperProducts & $W Y$ & BasicMaterials & Forestry\&WoodProducts & 0.5783 \\
\hline 30 & $N S M$ & Technology & Semiconductors & $T E R$ & Technology & Semiconductors & 0.6205 \\
\hline 31 & $M E R$ & Financial & InvestmentServices & $\overline{L E H}$ & Financial & InvestmentServices & 0.6225 \\
\hline 32 & $K R$ & Services & RetailGrocery & $A B S$ & Services & RetailGrocery & 0.6265 \\
\hline 33 & $H D$ & Services & RetailHomeImprovement & $L O W$ & Services & RetailHomeImprovement & 0.6305 \\
\hline 34 & $S W Y$ & Services & RetailGrocery & $A B S$ & Services & RetailGrocery & 0.6606 \\
\hline 35 & $P P G$ & BasicMaterials & ChemicalManifacturing & $E C$ & BasicMaterials & ChemicalManifacturing & 0.6627 \\
\hline 36 & $B H I$ & Energy & OilWellServ\&Equip & $S I I$ & Energy & OilWellServ\&Equip & 0.6727 \\
\hline 37 & $B N I$ & Transportation & Railroad & $C S X$ & Transportation & Railroad & 0.6767 \\
\hline 38 & WLP & Financial & InsAccidental\&Health & MME & Healthcare & $\mathbf{N} \backslash \mathbf{A}$ & 0.6767 \\
\hline 39 & $\mathrm{NSC}$ & Transportation & Railroad & $C S X$ & Transportation & Railroad & 0.6968 \\
\hline 40 & IP & BasicMaterials & Paper\&PaperProducts & TIN & Conglomerates & Conglomerates & 0.7048 \\
\hline 41 & $K R$ & Services & RetailGrocery & $S W Y$ & Services & RetailGrocery & 0.7129 \\
\hline 42 & WY & BasicMaterials & Forestry\&WoodProducts & TIN & Conglomerates & Conglomerates & 0.7169 \\
\hline 43 & GP & BasicMaterials & Paper\&PaperProducts & TIN & Conglomerates & Conglomerates & 0.7329 \\
\hline 44 & $P X$ & BasicMaterials & ChemicalManifacturing & $A P D$ & BasicMaterials & ChemicalManifacturing & 0.7771 \\
\hline 45 & $G C I$ & Services & Printing\&Publishing & $K R I$ & Services & Printing\&Publishing & 0.7992 \\
\hline 46 & $P G$ & ConsNonCycl & Personal\&HouseholdProducts & $C L$ & ConsNonCycl & Personal\&HouseholdProducts & 0.8072 \\
\hline 47 & $M R K$ & Healthcare & MajorDrugs & $B M Y$ & Healthcare & MajorDrugs & 0.8153 \\
\hline 48 & $C L$ & ConsNonCycl & Personal\&HouseholdProducts & $C L X$ & ConsNonCycl & Personal\&HouseholdProducts & 0.8333 \\
\hline 52 & $\overline{U C L}$ & Energy & Oil\&GasOperations & $A H C$ & Energy & Oil\&Gas - Integrated & 0.8775 \\
\hline 53 & $L T R$ & Financial & InsProp.\&Casualty & $J P$ & Financial & InsLife & 0.8876 \\
\hline 54 & $B L S$ & Services & CommunicationServices & $A T$ & Services & CommunicationServices & 0.8956 \\
\hline 55 & $C L$ & ConsNonCycl & Personal\&HouseholdProducts & $A V P$ & ConsNonCycl & Personal\&HouseholdProducts & 0.9016 \\
\hline 56 & $I T W$ & CapitalGood & Misc.CapitalGoods & $E T N$ & CapitalGood & Misc.CapitalGoods & 0.9116 \\
\hline 57 & $M R K$ & Healthcare & MajorDrugs & $A B T$ & Healthcare & MajorDrugs & 0.9157 \\
\hline 58 & $P P G$ & BasicMaterials & ChemicalManifacturing & $\mathrm{ROH}$ & BasicMaterials & Chemical - Plastic\&Rubber & 0.9378 \\
\hline 59 & $S P G$ & Services & RealEstateOperations & $E Q R$ & Services & RealEstateOperations & 0.9438 \\
\hline 60 & $C B$ & Financial & InsProp.\&Casualty & $J P$ & Financial & InsLife & 0.9478 \\
\hline 61 & $T X N$ & Technology & Semiconductors & $A D I$ & Technology & Semiconductors & 0.9558 \\
\hline 62 & ETN & CapitalGood & Misc.CapitalGoods & $\mathbf{P H}$ & BasicMaterials & Misc.FabricatedProducts & 0.9719 \\
\hline 63 & $A P A$ & Energy & Oil\&GasOperations & $A P C$ & Energy & Oil\&GasOperations & 0.9759 \\
\hline 64 & $U C L$ & Energy & Oil\&GasOperations & $K M G$ & Energy & Oil\&GasOperations & 0.9819 \\
\hline 65 & $P F E$ & Healthcare & MajorDrugs & $M R K$ & Healthcare & MajorDrugs & 0.99 \\
\hline 66 & $S P G$ & Services & RealEstateOperations & $G G P$ & Services & RealEstateOperations & 0.996 \\
\hline 67 & $A T$ & Services & CommunicationServices & $C T L$ & Services & CommunicationServices & 0.996 \\
\hline 68 & PPG & BasicMaterials & ChemicalManifacturing & TIN & Conglomerates & Conglomerates & 0.996 \\
\hline 69 & $C A T$ & CapitalGood & Constr.\&Agric.Machinery & $D E$ & CapitalGood & Constr.\&Agric.Machinery & 0.998 \\
\hline 70 & $A P A$ & Energy & Oil\&GasOperations & $B R$ & Energy & Oil\&GasOperations & 0.998 \\
\hline
\end{tabular}

commercial constructions" for families and firms (details have been retrieved from companies's Web pages).

Table 9 reports some of the most significant edges that are always selected by $P M F G$ s but not always selected by MSTs. Once more, we see clearly that most of these edges have both nodes belonging to the same sector and sub-sector, showing that the system of correlations is highly clustered. For instance, rows 11 and 38 are similar to row 7 of Table 7 and row 9 of Table 8. We find particularly interesting the edges involving Temple-Inland $(T I N)$ (rows 40, 42, 43, 68), from the Conglomerates sector, that is always linked to companies belonging to the sector of Basic Materials and subsectors Forestry, Wood, Paper and Chemical Products. Temple-Inland, indeed, engages in corrugated packaging and forest products (real estate and financial services businesses). It manufactures a range of building products including lumber, studs, gypsum wallboard, engineered wood siding and trim, fiberboard sheathing. 

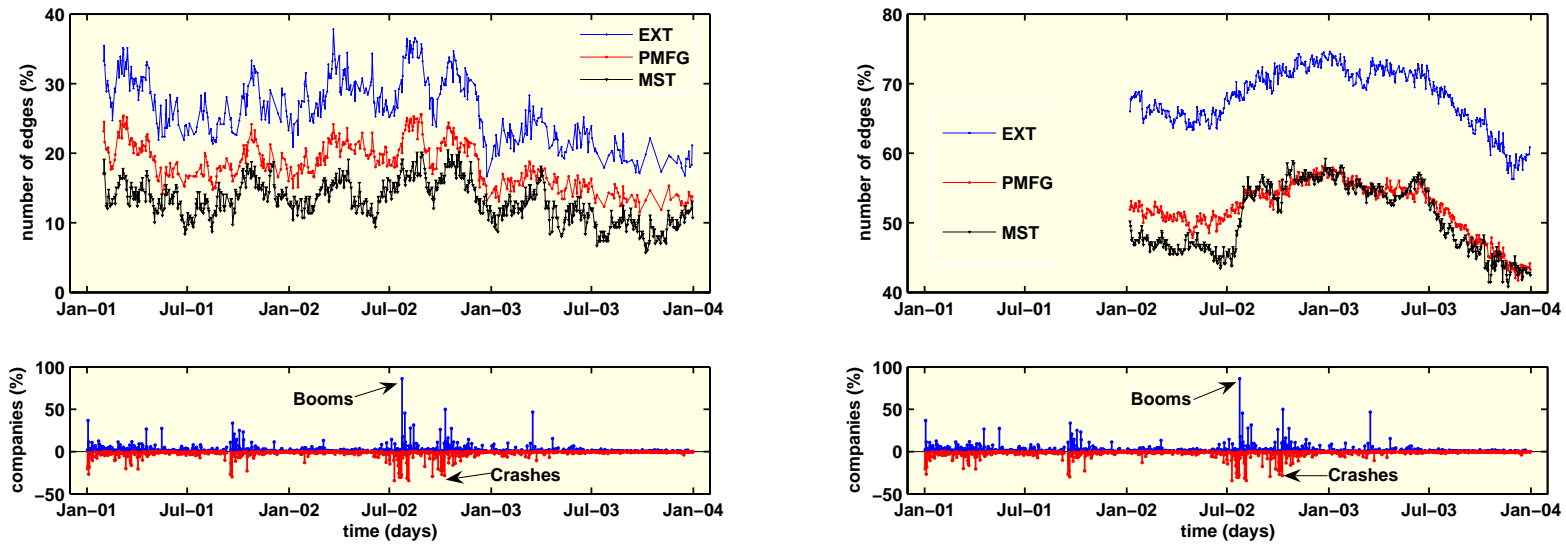

Figure 4. Percentages of persistent edges belonging (from top to bottom) to: graphs obtained by T1 expansion of $P M F G$; dynamical $\operatorname{PMFG}(t, \Delta t)$, dynamical $M S T(t, \Delta t)$. On the left: $\Delta t=21$; on the right: $\Delta t=251$. At the bottom, percentages of companies whose standardized return, at each time $t$, exceeds two standard deviations or falls below minus two standard deviations.

From Table 8 and Table 9, we see that the $P M F G$ procedure selects some especially high quality edges that are missing, always or most of the times, from the $M S T$.

\section{LONG RUN TIME PERSISTENCES FOR EDGES OF SUB-GRAPHS}

Onnela ${ }^{15}$ and Johnson ${ }^{16}$ introduced some interesting measures of survival for edges belonging to dynamical graphs: in particular they propose to calculate the common edges between $G(t+k)$ and $G(t)$ (single step survival ratio); or between $G(t+k), G(t+k-1), \ldots, G(t+1)$ and $G(t)$ (k multi-step survival ratio). These are short-run measures of persistence, weak in the former case; stronger, and rather restrictive, in the latter.

In this paper we have further considered the intersections between dynamical subgraphs and their corresponding static subgraphs. We have then calculated, for each $t$ and $\Delta t$, the number of common edges between dynamical $M S T(t, \Delta t)$ and the Static $M S T$ divided by the length of the $M S T$; the number of common edges between dynamical $P M F G(t, \Delta t)$ and the Static $P M F G$ divided by the length of the $P M F G$. As we can see in Figure 4, when $\Delta t=21$ days the dynamical $P M F G(t, \Delta t)$ seem to be more stable than the dynamical $\operatorname{MST}(t, \Delta t)$ and still slightly more stable also in the case $\Delta t=251$ days.

Following an insight from Ohlenbusch et al. ${ }^{4}$ and Aste et al. ${ }^{5}$ we have considered for each $t$ and $\Delta t$, all local $T 1$ elementary topological movements for all edges of the $P M F G$ s. A $T 1$ movement is an edge-switching process consisting in joining nodes $c$ and $d$ if and only if they are common neighbors of nodes $a$ and $b$, where $a$ and $b$ are already linked by an edge in the graph. After joining all such nodes, we obtain a new expanded graph that contains all possible evolutions of the original planar through local $T 1$ elementary topological movements. The procedure described for the planar graphs cannot be carried out for trees, since if two nodes have two common neighbors then there must be a cycle in the graph, so this cannot be a tree.

We find that the persistence of edges belonging to the new dynamical expanded planar graphs with respect to the static planar is higher than the others when $\Delta t=21$ days and sensitively higher when $\Delta t=251$ days.

\section{CONCLUSIONS AND FUTURE RESEARCH}

Financial systems are highly complex systems. Available data need to be filtered in order to be able to extract relevant and meaningful information out of an extremely huge amount of data.

In this paper we have shown that both $M S T$ and $P M F G$ reproduce pretty well the properties of the system and are structurally robust. They both select some particularly significant edges of the economic underlying system. We have seen that edges selected by both $M S T$ and $P M F G$ are impressively clustered within economic 
sectors and subsectors, with the $P M F G$ having a richer number of high-quality details on the financial system with respect to the MSTs.

We have introduced a new measure of survival for edges of a graph that catches their long-run persistence. We have found that the $P M F G$ seems to be slightly more persistent from a structural point of view, in the long-run, even in the case $\Delta t=251$ days when both subgraphs are particularly robust. We have seen that, if we expand the $P M F G$ by adding edges through local $T 1$ elementary topological movements, we obtain a graph that retains, in the most robust case, most of the edges belonging to the Static PMFG.

Further steps will be taken to investigate the robustness and the meaning of those edges that show high clustering power from an economic sectorial point of view.

\section{ACKNOWLEDGMENTS}

This work was partially supported by the ARC Discovery Projects DP0344004 (2003), DP0558183 (2005) and COST P10 "Physics of Risk" project.

F. Pozzi kindly acknowledges the Department of Public Economics and the Doctoral School of Economics of the University of Rome "La Sapienza" for a one-year research scholarship and for the authorization to spend this period at the ANU; he also acknowledges the hospitality of The Australian National University.

\section{REFERENCES}

1. J. C. Gower, G. J. S. Ross, "Minimum Spanning Trees and Single Linkage Cluster Analysis", Applied Statistics, 18/1, 54-64, 1969.

2. R. N. Mantegna, "Hierarchical structure in financial markets", EPJB, 11, 193-197, 1999.

3. M. Tumminello, T. Aste, T. Di Matteo and R. N. Mantegna, "A tool for filtering information in complex systems", PNAS, 102/30, 10421-10426, 2005.

4. H. M. Ohlenbusch, T. Aste, B. Dubertret, N. Rivier, "The topological structure of 2D disordered cellular systems", EPJB, 2, 211-220, 1998.

5. T. Aste, D. Sherrington, "Glass transition in self-organizing cellular patterns", J. Phys. A, 32, 7049-56, 1999.

6. J. Eisner, "State-of-the-art Algorithms for MSTs: A Tutorial Discussion", Manuscript, University of Pennsylvania, 1997.

7. B. Chazelle, "A Minimum Spanning Tree Algorithm with Inverse-Ackermann Type Complexity", Journal of the ACM (JACM), 47/6, 1028-1047, 2000.

8. A planar graph is a network that can be represented on an Euclidean plane with no intersections between edges.

9. T. Di Matteo, T. Aste, "How does the Eurodollar Interest Rate behave?", International Journal of Theoretical and Applied Finance, 5/1, 107-122, 2002.

10. T. Di Matteo, T. Aste, R. N. Mantegna, "An interest rates cluster analysis", Physica A, 339, 181-188, 2004.

11. T. Aste, T. Di Matteo, M. Tumminello, R. N. Mantegna, "Correlation filtering in financial time series", Proceedings-SPIE The International Society For Optical Engineering, 5848, 100-109, 2005.

12. T. Di Matteo, T. Aste, S. T. Hyde, S. Ramsden, "Interest rates hierarchical structure", Physica A, 355, 21-33, 2005.

13. T. Aste, T. Di Matteo, "Dynamical networks from correlations", Physica A, 370, 156-161, 2006.

14. T. Di Matteo, T. Aste, "Extracting the correlation structure by means of planar embedding", ProceedingsSPIE The International Society For Optical Engineering, 6039, 60390P-1, 2006.

15. J.-P. Onnela, "Taxonomy of Financial Assets", M.Sc. Thesis, Department of Electrical and Communications Engineering, Helsinki University of Technology, 2002.

16. M. McDonald, O. Suleman, S. Williams, S. Howison, N. F. Johnson, "Detecting a currency's dominance or dependence using foreign exchange network trees", Physical Review E 72, 046106, 2005.

17. T. Aste, T. Di Matteo, S. T. Hyde, "Complex networks on hyperbolic surfaces", Physica A, 346, 20-26, 2005.

18. M. Tumminello, T. Aste, T. Di Matteo, R. N. Mantegna, "Correlation based networks of equity returns sampled at different time horizons", EPJB, 55, 209-217, 2007. 